\title{
Computing of Mutual Admittance between Two Circumferential Slots on Cylindrical Waveguide
}

\author{
Ali Khayati' ${ }^{1}$ Amir Amirabadi², Behnam Rajabi ${ }^{3}$ \\ ${ }^{1}$ Islamic Azad University Parand Branch, Parand, Iran \\ ${ }^{2}$ Islamic Azad University South Tehran Branch, Tehran, Iran \\ ${ }^{3}$ Islamic Azad University Tuyserkan Branch, Tuyserkan, Iran \\ Email: Khaiiaty@gmail.com, a_amirabadi@azad.ac.ir,b.rajabi@tuyiau.ac.ir
}

How to cite this paper: Khayati, A., Amirabadi, A. and Rajabi, B. (2017) Computing of Mutual Admittance between Two Circumferential Slots on Cylindrical Waveguide. Open Journal of Antennas and Propagation, 5, 188-199.

https://doi.org/10.4236/ojapr.2017.54015

Received: September 5, 2017

Accepted: December 18, 2017

Published: December 21, 2017

Copyright (C) 2017 by authors and Scientific Research Publishing Inc. This work is licensed under the Creative Commons Attribution International License (CC BY 4.0).

http://creativecommons.org/licenses/by/4.0/

\begin{abstract}
This paper studies radiation from circumferential slots on cylindrical waveguide by Poynting's vector method. It can help us to find mutual admittance between two circumferential slots in an antenna array. The main advantage of Poynting's vector method is its accurate convergence to compute mutual admittance between two circumferential slots. The importance of this matter will be more salient while we want to compare it with other mutual admittances and also use it to optimize an antenna array.
\end{abstract}

\section{Keywords}

Circumferential Slot, Cylindrical Waveguide, Poynting's Vector, Mutual Admittance

\section{Introduction}

Electromagnetic radiation from an aperture waveguide is one kind of classical and practical problems which has been considered for several years. Because of cylindrical waveguide and conformal antenna applications, same as aperture waveguide radiation, electromagnetic radiation from slots on cylindrical waveguide is a prominent problem. To have a better analysis, desired pattern and also to have a proper matching in input, electromagnetic radiation and mutual coupling between slots present an equation to compute mutual admittance between apertures on cylindrical waveguide.

To compute mutual admittance, apart from aperture shape on cylindrical waveguide, there are different methods which have been used but generally they are divided into two general categories:

- The methods which are based on reciprocity (reaction) theorem that involve 
modal solution [1]-[7] and surface ray or GTD-Solutions [8]

- The methods which are based on Poynting's vector [9].

In modal analysis method we need to have several mode combinations to obtain an accurate approach, if the mode numbers aren't sufficient, analysis accuracy will be decreased. To have more mode numbers, radius of cylinder must be increased, so this method is not useful for such a problem which the mode numbers are insufficient. If the radius of cylinder be one or two times bigger than wavelength units, surface ray method or GTD-Solution will be helpful. The method which is used in here is based on Poynting's vector method due to its advantages for circumferential slot array designing.

This method has much more conformity with cylindrical waveguide to reach convergence. The method which is used in here was suggested by Hill [10] to find input impedance for dipole antenna. It also can be used to find mutual impedance between axial slots on cylindrical waveguide [11]. So Poynting's vector method can be indicated such as following relation:

$$
Y_{12}=\frac{1}{V_{2} V_{1}^{*}} \int_{c} \int_{0}^{2 \pi} \boldsymbol{E}_{2} \times \boldsymbol{H}_{1}^{*} \cdot \hat{n} r^{2} \sin \theta \mathrm{d} \theta \mathrm{d} \varphi
$$

Which $Y_{12}$ is mutual impedance between two slots number 1 and $2, \boldsymbol{E}_{2}$ is far electrical radiated field which is emerged from $V_{2}$ and $\boldsymbol{H}_{1}^{*}$ is far conjugate magnetic field which is emerged by $V_{1}$ in (1).

\section{Computing Mutual Admittance}

Far field radiation from circumferential slots [12] whereas voltage has cosine distribution can be written as follows:

$$
\begin{gathered}
E_{i \theta}=\frac{V_{i} \alpha_{i} \mathrm{e}^{-j k r_{i}}}{j \pi r_{i} \sin (\theta)} \sum_{n=-\infty}^{\infty} \frac{j^{n} \cos \left(\frac{n \alpha_{i}}{2}\right) \mathrm{e}^{j n\left(\varphi-\varphi_{i}\right)}}{\left[\pi^{2}-\left(n \alpha_{i}\right)^{2}\right] H_{n}^{(2)}(k a \sin (\theta))} \operatorname{sinc}\left(\frac{W k \cos (\theta)}{2 \pi}\right) \\
E_{i \varphi}=-\frac{V_{i} \alpha_{i} \mathrm{e}^{-j k r_{i}}}{\pi r_{i} k a \sin (\theta)} \cot (\theta) \sum_{n=-\infty}^{\infty} \frac{m j^{m} \cos \left(\frac{m \alpha_{i}}{2}\right) \mathrm{e}^{j m\left(\varphi-\varphi_{i}\right)}}{\left[\pi^{2}-\left(m \alpha_{i}\right)^{2}\right] H_{m}^{(2)}(a \sin (\theta))} \\
\cdot \operatorname{sinc}\left(\frac{W k \cos (\theta)}{2 \pi}\right) \quad E_{\varphi}=\frac{E_{\varphi}}{\eta}, H_{\theta}=-\frac{E^{\prime}}{\eta}
\end{gathered}
$$

where $W=3.5 \mathrm{~mm}$ and $k=\frac{2 \pi}{\lambda}$ which $\lambda$ is wavelength of propagated wave with vacuum dielectric, $a$ is radius of cylinder and $V_{i}$ is voltage which is emerged from distributed field on $i$ th slot and $\alpha_{i}$ is slot length depending on degree. In here $r_{i} \approx r-z_{i} k \cos (\theta)$ and $\varphi_{i}$ is offset of $i^{\prime}$ th slot according to $x$ axis, $\eta$ is intrinsic impedance of environment and $r, \theta$ and $\varphi$ are spherical coordinate (Figure 1). 
$H_{m}^{(2) '}($.$) is derivative of second type of Henkel function. In above relations$ time-dependent function ( $\mathrm{e}^{\mathrm{j} \omega t}$ ) is neglected. Now, above relations must be considered for finding mutual impedance so according to (1) the result of $\boldsymbol{E}_{2} \times \boldsymbol{H}_{1}^{*}$ can be presented as follows:

$$
\begin{gathered}
\boldsymbol{E}_{2}=E_{2 \theta} \hat{a}_{\theta}+E_{2 \varphi} \hat{a}_{\varphi} \\
\boldsymbol{H}_{1}=H_{1 \theta} \hat{a}_{\theta}+H_{1 \varphi} \hat{a}_{\varphi} \\
\boldsymbol{E}_{2} \times \boldsymbol{H}_{1}^{*}=\left(E_{2 \theta} \hat{a}_{\theta}+E_{2 \varphi} \hat{a}_{\varphi}\right) \times\left(H_{1 \theta} \hat{a}_{\theta}+H_{1 \varphi} \hat{a}_{\varphi}\right)^{*} \\
=\left(E_{2 \theta} H_{1 \varphi}^{*}-E_{2 \varphi} H_{1 \theta}^{*}\right) \hat{a}_{r}=\left(E_{2 \theta} \frac{E_{1 \theta}^{*}}{\eta}+E_{2 \varphi} \frac{E_{1 \varphi}^{*}}{\eta}\right) \hat{a}_{r}
\end{gathered}
$$

Integration path $\mathrm{C}$ can be shown as Figure 2:

Suppose that in cylindrical coordination system, slot position of I and II are $\left(a, \varphi_{2}, z_{2}\right)$ and $\left(a, \varphi_{1}, z_{1}\right)$.

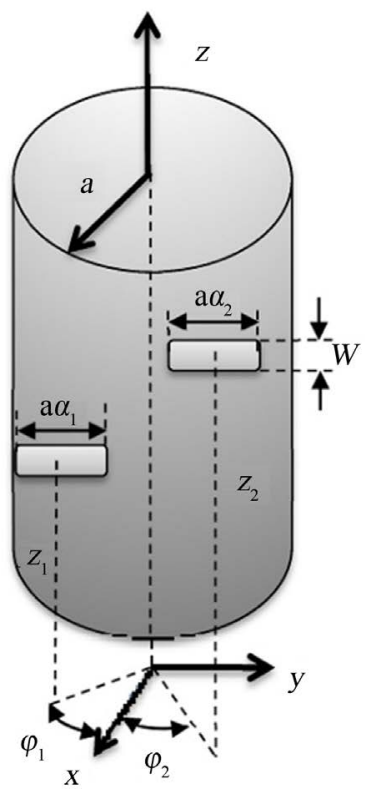

Figure 1. Two circumferential slots on cylindrical waveguide with their coordination.

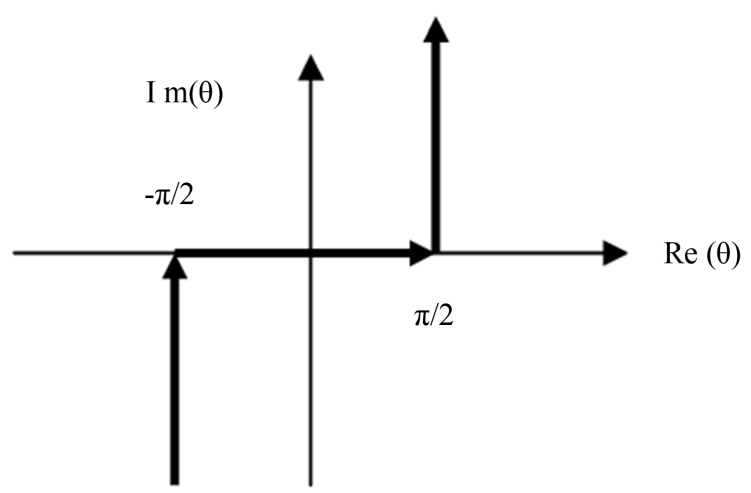

Figure 2. Integration path C. 
According to above coordination system, electric and magnetic far field radiation is:

$$
E_{1 \theta}=\frac{V_{1} \alpha_{1} \mathrm{e}^{-j k r_{1}}}{j \pi r_{1} \sin (\theta)} \sum_{n=-\infty}^{\infty} \frac{j^{n} \cos \left(\frac{n \alpha_{1}}{2}\right) \mathrm{e}^{j n\left(\varphi-\varphi_{1}\right)}}{\left[\pi^{2}-\left(n \alpha_{1}\right)^{2}\right] H_{n}^{(2)}(k a \sin (\theta))} \operatorname{sinc}\left(\frac{W k \cos (\theta)}{2 \pi}\right)
$$

Which $r_{1} \approx r-z_{1} k \cos (\theta)$. For $E_{2 \varphi}$ we have:

$$
\begin{aligned}
E_{2 \varphi}= & -\frac{V_{2} \alpha_{2} \mathrm{e}^{-j k r_{2}}}{\pi r_{2} k a \sin (\theta)} \cot (\theta) \sum_{m=-\infty}^{\infty} \frac{m j^{m} \cos \left(\frac{m \alpha_{2}}{2}\right) \mathrm{e}^{j m\left(\varphi-\varphi_{2}\right)}}{\left[\pi^{2}-\left(m \alpha_{1}\right)^{2}\right] H_{m}^{(2) \prime}(a \sin (\theta))} \\
& \cdot \operatorname{sinc}\left(\frac{W k \cos (\theta)}{2 \pi}\right)
\end{aligned}
$$

That $r_{2} \approx r-z_{2} k \cos (\theta)$.

Now the result of $\boldsymbol{E}_{2} \times \boldsymbol{H}_{1}^{*}$ must be defined, so first of all the term of $E_{2 \theta} \frac{E_{1 \theta}^{*}}{\eta}$ must be computed as follow:

$$
\begin{gathered}
E_{2 \theta}=\frac{V_{2} \alpha_{2} \mathrm{e}^{-j k r_{2}}}{j \pi r_{2} \sin (\theta)} \sum_{n=-\infty}^{\infty} \frac{j^{n} \cos \left(\frac{n \alpha_{2}}{2}\right) \mathrm{e}^{j n\left(\varphi-\varphi_{2}\right)}}{\left[\pi^{2}-\left(n \alpha_{2}\right)^{2}\right] H_{n}^{(2)}(k a \sin (\theta))} \sin c\left(\frac{W k \cos (\theta)}{2 \pi}\right)(10) \\
E_{1 \theta}^{*}=\frac{V_{1}^{*} \alpha_{1} \mathrm{e}^{j k k_{1}}}{-j \pi r_{1} \sin (\theta)} \sum_{n=-\infty}^{\infty} \frac{(-j)^{n} \cos \left(\frac{n \alpha_{1}}{2}\right) \mathrm{e}^{-j n\left(\varphi-\varphi_{1}\right)}}{\left[\pi^{2}-\left(n \alpha_{1}\right)^{2}\right] H_{n}^{(2) *}(k a \sin (\theta))} \operatorname{sinc}\left(\frac{W k \cos (\theta)}{2 \pi}\right) \\
\frac{E_{2 \theta} E_{1 \theta}^{*}}{\eta}=\frac{V_{2} V_{1}^{*} \alpha_{1} \alpha_{2} \mathrm{e}^{-j k\left(r_{2}-r_{1}\right)}}{\eta \pi^{2} r_{1} r_{2} \sin ^{2}(\theta)} \cdot \sum_{m=-\infty}^{\infty} \sum_{m=-\infty}^{\infty} \frac{j^{m-n} \cos \left(\frac{m \alpha_{2}}{2}\right) \cos \left(\frac{n \alpha_{1}}{2}\right)}{\left.\pi^{2}-\left(m \alpha_{2}\right)^{2}\right]\left[\pi^{2}-\left(n \alpha_{1}\right)^{2}\right]} \\
\frac{\mathrm{e}^{j m\left(\varphi-\varphi_{2}\right)} \mathrm{e}^{-j n\left(\varphi-\varphi_{1}\right)}}{H_{m}^{(2)}(k a \sin (\theta)) H_{n}^{(2) *}(k a \sin (\theta))} \cdot \operatorname{sinc}^{2}\left(\frac{W k \cos (\theta)}{2 \pi}\right)
\end{gathered}
$$

Then for $E_{2 \varphi} \frac{E_{1 \varphi}^{*}}{\eta}$ we have:

$$
\begin{aligned}
E_{1 \varphi}^{*}= & -\frac{V_{1}^{*} \alpha_{1} \mathrm{e}^{j k r_{1}}}{\pi r_{1} k a \sin (\theta)} \cot (\theta) \sum_{n=-\infty}^{\infty} \frac{n(-j)^{n} \cos \left(\frac{n \alpha_{1}}{2}\right) \mathrm{e}^{-j n\left(\varphi-\varphi_{1}\right)}}{\left[\pi^{2}-\left(n \alpha_{1}\right)^{2}\right] H_{n}^{(2))^{* *}}(k a \sin (\theta))} \\
& \cdot \operatorname{sinc}\left(\frac{W k \cos (\theta)}{2 \pi}\right) \\
E_{2 \varphi}= & -\frac{V_{2} \alpha_{2} \mathrm{e}^{-j k r_{2}}}{\pi r_{2} k a \sin (\theta)} \cot (\theta) \sum_{m=-\infty}^{\infty} \frac{m j^{m} \cos \left(\frac{m \alpha_{2}}{2}\right) \mathrm{e}^{j m\left(\varphi-\varphi_{2}\right)}}{\left[\pi^{2}-\left(m \alpha_{2}\right)^{2}\right] H_{m}^{(2) \prime}(k a \sin (\theta))} \\
& \cdot \operatorname{sinc}\left(\frac{W k \cos (\theta)}{2 \pi}\right)
\end{aligned}
$$




$$
\begin{aligned}
& E_{2 \varphi} \frac{E_{1 \varphi}^{*}}{\eta}=\frac{V_{1}^{*} V_{2} \alpha_{2} \alpha_{1} \mathrm{e}^{-j k\left(r_{2}-r_{1}\right)}}{\pi^{2} r_{1} r_{2}(k a \sin (\theta))^{2}}(\cot (\theta))^{2} \\
& \cdot \sum_{m=-\infty}^{\infty} \sum_{n=-\infty}^{\infty} \frac{m n j^{m-n} \cos \left(\frac{n \alpha_{1}}{2}\right) \cos \left(\frac{m \alpha_{2}}{2}\right) \mathrm{e}^{j m\left(\varphi-\varphi_{2}\right)} \mathrm{e}^{-j n\left(\varphi-\varphi_{1}\right)}}{\left[\pi^{2}-\left(n \alpha_{1}\right)^{2}\right]\left[\pi^{2}-\left(m \alpha_{2}\right)^{2}\right] H_{m}^{(2) \prime}(k a \sin (\theta)) H_{n}^{(2)^{\prime *}}(k a \sin (\theta))} \\
& \cdot \operatorname{sinc}^{2}\left(\frac{W k \cos (\theta)}{2 \pi}\right)
\end{aligned}
$$

To simplify integral computation, it must be divided in to two parts then compute any part separately and finally the results must be added:

$$
\begin{aligned}
Y_{12}= & \frac{1}{V_{2} V_{1}^{*}} \int_{c} \int_{0}^{2 \pi} \boldsymbol{E}_{2} \times \boldsymbol{H}_{1}^{*} \cdot \hat{n} r^{2} \sin \theta \mathrm{d} \theta \mathrm{d} \varphi \\
= & \frac{1}{V_{2} V_{1}^{*}} \int_{c} \int_{0}^{2 \pi}\left(E_{2 \theta} \frac{E_{1 \theta}^{*}}{\eta}+E_{2 \varphi} \frac{E_{1 \varphi}^{*}}{\eta}\right) \hat{a}_{r} \cdot \hat{a}_{r} r^{2} \sin \theta \mathrm{d} \theta \mathrm{d} \varphi \\
= & \frac{1}{V_{2} V_{1}^{*}} \int_{c} \int_{0}^{2 \pi}\left(E_{2 \theta} \frac{E_{1 \theta}^{*}}{\eta}\right) r^{2} \sin \theta \mathrm{d} \theta \mathrm{d} \varphi \\
& +\frac{1}{V_{2} V_{1}^{*}} \int_{c} \int_{0}^{2 \pi}\left(E_{2 \varphi} \frac{E_{1 \varphi}^{*}}{\eta}\right) r^{2} \sin \theta \mathrm{d} \theta \mathrm{d} \varphi=Y_{12}^{1}+Y_{12}^{2} \\
Y_{12}^{1}= & \frac{1}{V_{2} V_{1}^{*}} \int_{c} \int_{0}^{2 \pi}\left(E_{2 \theta} \frac{E_{1 \theta}^{*}}{\eta}\right) r^{2} \sin \theta \mathrm{d} \theta \mathrm{d} \varphi \\
Y_{12}^{2}= & \frac{1}{V_{2} V_{1}^{*}} \int_{c} \int_{0}^{2 \pi}\left(E_{2 \varphi} \frac{E_{1 \varphi}^{*}}{\eta}\right) r^{2} \sin \theta \mathrm{d} \theta \mathrm{d} \varphi
\end{aligned}
$$

In another hand to compute $Y_{12}^{1}$ we have:

$$
\begin{aligned}
Y_{12}^{1}= & \frac{1}{V_{2} V_{1}^{*}} \int_{c} \int_{0}^{2 \pi}\left(E_{2 \theta} \frac{E_{1 \theta}^{*}}{\eta}\right) r^{2} \sin \theta \mathrm{d} \theta \mathrm{d} \varphi \\
= & \frac{1}{V_{2} V_{1}^{*}} \int_{c} \int_{0}^{2 \pi} \frac{V_{2} V_{1}^{*} \alpha_{1} \alpha_{2} \mathrm{e}^{-j k\left(r_{2}-r_{1}\right)}}{\eta \pi^{2} r_{1} r_{2} \sin ^{2}(\theta)} \sum_{m=-\infty}^{\infty} \sum_{n=-\infty}^{\infty} \frac{j^{m-n} \cos \left(\frac{m \alpha_{2}}{2}\right) \cos \left(\frac{n \alpha_{1}}{2}\right)}{\left[\pi^{2}-\left(m \alpha_{2}\right)^{2}\right]\left[\pi^{2}-\left(n \alpha_{1}\right)^{2}\right]} \\
& \cdot \frac{\mathrm{e}^{j m\left(\varphi-\varphi_{2}\right)} \mathrm{e}^{-j n\left(\varphi-\varphi_{1}\right)}}{H_{m}^{(2)}(k a \sin (\theta)) H_{n}^{(2) *}(k a \sin (\theta))} \operatorname{sinc}^{2}\left(\frac{W k \cos (\theta)}{2 \pi}\right) r^{2} \sin \theta \mathrm{d} \theta \mathrm{d} \varphi
\end{aligned}
$$

According to asymmetry of integration path and term of $Y_{12}^{1}$, so integration path can be assumed as:

So:

$$
\begin{aligned}
Y_{12}^{1}= & \frac{1}{V_{2} V_{1}^{*}} \int_{c} \int_{0}^{2 \pi}\left(E_{2 \theta} \frac{E_{1 \theta}^{*}}{\eta}\right) r^{2} \sin \theta \mathrm{d} \theta \mathrm{d} \varphi \\
= & \frac{2 \alpha_{1} \alpha_{2}}{\eta \pi^{2} r_{1} r_{2}} \int_{c} \int_{0}^{2 \pi} \frac{\mathrm{e}^{-j k\left(r_{2}-r_{1}\right)}}{\sin ^{2}(\theta)} \sum_{m=-\infty}^{\infty} \sum_{n=-\infty}^{\infty} \frac{j^{m-n} \cos \left(\frac{m \alpha_{2}}{2}\right) \cos \left(\frac{n \alpha_{1}}{2}\right)}{\left[\pi^{2}-\left(m \alpha_{2}\right)^{2}\right]\left[\pi^{2}-\left(n \alpha_{1}\right)^{2}\right]} \\
& \cdot \frac{\mathrm{e}^{j m\left(\varphi-\varphi_{2}\right)} \mathrm{e}^{-j n\left(\varphi-\varphi_{1}\right)}}{H_{m}^{(2)}(k a \sin (\theta)) H_{n}^{(2) *}(k a \sin (\theta))} \operatorname{sinc}^{2}\left(\frac{W k \cos (\theta)}{2 \pi}\right) r^{2} \sin \theta \mathrm{d} \theta \mathrm{d} \varphi
\end{aligned}
$$


After substitution of $r_{1}$ and $r_{2}$ in (20) and according to even symmetry with respect to $\varphi$, integral can be simplify as:

$$
\begin{aligned}
Y_{12}^{1}= & \frac{2 \alpha_{1} \alpha_{2}}{\eta \pi^{2}} \int_{c} \int_{0}^{2 \pi} \frac{\mathrm{e}^{-j k \cos (\theta)\left(z_{2}-z_{1}\right)}}{\sin ^{2}(\theta)} \sin \theta \sum_{m=0}^{\infty} \sum_{n=0}^{\infty} \frac{\varepsilon_{m} \varepsilon_{n} j^{m-n} \cos \left(\frac{m \alpha_{2}}{2}\right) \cos \left(\frac{n \alpha_{1}}{2}\right)}{\left[\pi^{2}-\left(m \alpha_{2}\right)^{2}\right]\left[\pi^{2}-\left(n \alpha_{1}\right)^{2}\right]} \\
& \cdot \frac{\cos \left(m\left(\varphi-\varphi_{2}\right)\right) \cos \left(n\left(\varphi-\varphi_{1}\right)\right)}{H_{m}^{(2)}(k a \sin (\theta)) H_{n}^{(2) *}(k a \sin (\theta))} \operatorname{sinc}^{2}\left(\frac{W k \cos (\theta)}{2 \pi}\right) \mathrm{d} \theta \mathrm{d} \varphi
\end{aligned}
$$

Which:

$$
\varepsilon_{m}= \begin{cases}1 & m=0 \\ 2 & m \neq 0\end{cases}
$$

if $m=n$, term of $\cos \left(m\left(\varphi-\varphi_{2}\right)\right) \cos \left(n\left(\varphi-\varphi_{1}\right)\right)$ is an orthogonal function and it has definite values.

So integral can be simplified such as following relations:

$$
\begin{aligned}
Y_{12}^{1}= & \frac{2 \alpha_{1} \alpha_{2}}{\eta \pi^{2}} \int_{c} \int_{0}^{2 \pi} \frac{\mathrm{e}^{-j k \cos (\theta)\left(z_{2}-z_{1}\right)}}{\sin ^{2}(\theta)} \sin \theta \sum_{m=0}^{\infty} \sum_{n=0}^{\infty} \frac{\varepsilon_{n} \varepsilon_{n} \cos \left(\frac{n \alpha_{2}}{2}\right) \cos \left(\frac{n \alpha_{1}}{2}\right)}{\left[\pi^{2}-\left(n \alpha_{2}\right)^{2}\right]\left[\pi^{2}-\left(n \alpha_{1}\right)^{2}\right]} \\
& \frac{\cos \left(n\left(\varphi-\varphi_{2}\right)\right) \cos \left(n\left(\varphi-\varphi_{1}\right)\right)}{H_{n}^{(2)}(k a \sin (\theta)) H_{n}^{(2) *}(k a \sin (\theta))} \operatorname{sinc}^{2}\left(\frac{W k \cos (\theta)}{2 \pi}\right) \mathrm{d} \theta \mathrm{d} \varphi
\end{aligned}
$$

Above relations can be simplified more, if integral be found in term of following relations:

$$
\begin{aligned}
Y_{12}^{1}= & \frac{4 \alpha_{1} \alpha_{2}}{\eta \pi} \int_{c} \frac{\mathrm{e}^{-j k \cos (\theta)\left(z_{2}-z_{1}\right)}}{\sin ^{2}(\theta)} \sin \theta \cdot \sum_{n=0}^{\infty} \frac{\varepsilon_{n} \cos \left(\frac{n \alpha_{2}}{2}\right) \cos \left(\frac{n \alpha_{1}}{2}\right)}{\left[\pi^{2}-\left(n \alpha_{2}\right)^{2}\right]\left[\pi^{2}-\left(n \alpha_{1}\right)^{2}\right]} \\
& \cdot \frac{\cos \left(n\left(\varphi_{1}-\varphi_{2}\right)\right)}{\left|H_{n}^{(2)}(k a \sin (\theta))\right|} \operatorname{sinc}^{2}\left(\frac{W k \cos (\theta)}{2 \pi}\right) \mathrm{d} \theta
\end{aligned}
$$

The integral can be divided to imaginary and real parts.

For real part we have:

$$
k_{\mathrm{z}}=k \cos (\theta), \mathrm{d} k_{\mathrm{z}}=-k \sin \theta \mathrm{d} \theta, \theta=\left[0, \frac{\pi}{2}\right] \rightarrow k_{\mathrm{z}}=[k, 0]
$$

So result can be shown as follows:

$$
\begin{aligned}
Y_{12}^{1}= & \frac{4 \alpha_{1} \alpha_{2} k}{\eta \pi} \int_{0}^{k} \frac{\mathrm{e}^{-j k_{z}\left(z_{2}-z_{1}\right)}}{k^{2}-k_{z}^{2}} \sum_{n=0}^{\infty} \frac{\varepsilon_{n} \cos \left(\frac{n \alpha_{2}}{2}\right) \cos \left(\frac{n \alpha_{1}}{2}\right)}{\left[\pi^{2}-\left(n \alpha_{2}\right)^{2}\right]\left[\pi^{2}-\left(n \alpha_{1}\right)^{2}\right]} \\
& \cdot \frac{\cos \left(n\left(\varphi_{1}-\varphi_{2}\right)\right)}{\left|H_{n}^{(2)}\left(a \sqrt{k^{2}-k_{z}^{2}}\right)\right|} \operatorname{sinc}^{2}\left(\frac{W k_{z}}{2 \pi}\right) \mathrm{d} k_{z}
\end{aligned}
$$

Now we consider imaginary part, for new path can be shown: 


$$
\begin{gathered}
k_{z}=k \cos (\theta)=k \cos (\operatorname{Re}(\theta)+j \operatorname{Im}(\theta)) \\
=k[\cos (\operatorname{Re}(\theta)) \cosh (\operatorname{Im}(\theta))-\sin (\operatorname{Re}(\theta)) \sinh (\operatorname{Im}(\theta))] \\
\operatorname{Re}(\theta)=\frac{\pi}{2}, \operatorname{Im}(\theta)=[0, \infty] \Rightarrow k_{\mathrm{z}}=[0,-j \infty]
\end{gathered}
$$

It must be mentioned that $k_{z}=-j k_{z}^{\prime}, k_{z}^{\prime}=[0, \infty]$ so:

$$
\begin{aligned}
Y_{12}^{1}= & \frac{j 4 \alpha_{1} \alpha_{2} k}{\eta \pi} \int_{0}^{\infty} \frac{\mathrm{e}^{-k_{z}^{\prime}\left(z_{2}-z_{1}\right)}}{k^{2}+k_{z}^{\prime 2}} \sum_{n=0}^{\infty} \frac{\varepsilon_{n} \cos \left(\frac{n \alpha_{2}}{2}\right) \cos \left(\frac{n \alpha_{1}}{2}\right)}{\left[\pi^{2}-\left(n \alpha_{2}\right)^{2}\right]\left[\pi^{2}-\left(n \alpha_{1}\right)^{2}\right]} \\
& \cdot \frac{\cos \left(n\left(\varphi_{1}-\varphi_{2}\right)\right)}{\mid H_{n}^{(2)}\left(a \sqrt{k^{2}+k_{z}^{\prime 2}}\right)^{2}} \sinh ^{2}\left(\frac{W k_{z}^{\prime}}{2 \pi}\right) \mathrm{d} k_{z}^{\prime}
\end{aligned}
$$

where:

$$
\operatorname{sinch}\left(\frac{W k_{z}^{\prime}}{2 \pi}\right)=\frac{\sinh \left(\frac{W k_{z}^{\prime}}{2}\right)}{\frac{W k_{z}^{\prime}}{2}}
$$

Same as $Y_{12}$ we try to find $Y_{12}^{2}$ so:

$$
\begin{aligned}
& Y_{12}^{2}=\frac{1}{V_{2} V_{1}^{*}} \int_{c} \int_{0}^{2 \pi}\left(E_{2 \varphi} \frac{E_{1 \varphi}^{*}}{\eta}\right) r^{2} \sin \theta \mathrm{d} \theta \mathrm{d} \varphi \\
& E_{2 \varphi} \frac{E_{1 \varphi}^{*}}{\eta}=\frac{V_{1}^{*} V_{2} \alpha_{2} \alpha_{1} \mathrm{e}^{-j k\left(r_{2}-r_{1}\right)}}{\eta \pi^{2} r_{1} r_{2}(k a \sin (\theta))^{2}}(\cot (\theta))^{2} \sum_{m=-\infty}^{\infty} \sum_{m=-\infty}^{\infty} \frac{m n j^{m-n} \cos \left(\frac{n \alpha_{1}}{2}\right) \cos \left(\frac{m \alpha_{2}}{2}\right)}{\left[\pi^{2}-\left(n \alpha_{1}\right)^{2}\right]\left[\pi^{2}-\left(m \alpha_{2}\right)^{2}\right]} \\
& \cdot \frac{\mathrm{e}^{j m\left(\varphi-\varphi_{2}\right)} \mathrm{e}^{-j n\left(\varphi-\varphi_{1}\right)}}{H_{n}^{(2)^{\prime *}}(k a \sin (\theta)) H_{m}^{(2)^{\prime}}(k a \sin (\theta))} \operatorname{sinc}^{2}\left(\frac{W k \cos (\theta)}{2 \pi}\right) \\
& Y_{12}^{2}=\frac{1}{V_{2} V_{1}^{*}} \int_{c} \int_{0}^{2 \pi} \frac{V_{1}^{*} V_{2} \alpha_{2} \alpha_{1} \mathrm{e}^{-j k\left(r_{2}-r_{1}\right)}}{\pi^{2} r_{1} r_{2}(k a \sin (\theta))^{2}}(\cot (\theta))^{2} \\
& \cdot \sum_{m=-\infty}^{\infty} \sum_{n=-\infty}^{\infty} \frac{m n j^{m-n} \cos \left(\frac{n \alpha_{1}}{2}\right) \cos \left(\frac{m \alpha_{2}}{2}\right) \mathrm{e}^{j m\left(\varphi-\varphi_{2}\right)} \mathrm{e}^{-j n\left(\varphi-\varphi_{1}\right)}}{\left[\pi^{2}-\left(n \alpha_{1}\right)^{2}\right]\left[\pi^{2}-\left(m \alpha_{2}\right)^{2}\right] H_{m}^{(2) '}(k a \sin (\theta)) H_{n}^{(2)^{\prime *}}(k a \sin (\theta))} \\
& \text {. } \operatorname{sinc}^{2}\left(\frac{W k \cos (\theta)}{2 \pi}\right) r^{2} \sin \theta \mathrm{d} \theta \mathrm{d} \varphi \\
& \Rightarrow Y_{12}^{2}=\frac{\alpha_{2} \alpha_{1}}{\pi^{2} r_{1} r_{2}} \int_{c} \int_{0}^{2 \pi} \frac{\mathrm{e}^{-j k\left(r_{2}-r_{1}\right)}}{(k a \sin (\theta))^{2}}(\cot (\theta))^{2} \sum_{m=-\infty}^{\infty} \sum_{n=-\infty}^{\infty} \frac{m n j^{m-n} \cos \left(\frac{n \alpha_{1}}{2}\right) \cos \left(\frac{m \alpha_{2}}{2}\right)}{\left[\pi^{2}-\left(n \alpha_{1}\right)^{2}\right]\left[\pi^{2}-\left(m \alpha_{2}\right)^{2}\right]} \\
& \cdot \frac{\mathrm{e}^{j m\left(\varphi-\varphi_{2}\right)} \mathrm{e}^{-j n\left(\varphi-\varphi_{1}\right)}}{H_{n}^{(2))^{* *}}(k a \sin (\theta)) H_{m}^{(2) \prime}(k a \sin (\theta))} \operatorname{sinc}^{2}\left(\frac{W k \cos (\theta)}{2 \pi}\right) r^{2} \sin \theta \mathrm{d} \theta \mathrm{d} \varphi
\end{aligned}
$$

After substitution and by following above steps, the result will be as (29): 


$$
\begin{gathered}
Y_{12}^{2}=\frac{2 \alpha_{2} \alpha_{1}}{\eta \pi^{2}} \int_{c} \int_{0}^{2 \pi} \frac{\mathrm{e}^{-j k \cos (\theta)\left(z_{2}-z_{1}\right)}}{(k a \sin (\theta))^{2}}(\cot (\theta))^{2} \sum_{m=1 n=1}^{\infty} \sum^{\infty} \frac{m n j^{m-n} \cos \left(\frac{n \alpha_{1}}{2}\right) \cos \left(\frac{m \alpha_{2}}{2}\right)}{\left[\pi^{2}-\left(n \alpha_{1}\right)^{2}\right]\left[\pi^{2}-\left(m \alpha_{2}\right)^{2}\right]} \\
\cdot \frac{\mathrm{e}^{j m\left(\varphi-\varphi_{2}\right)} \mathrm{e}^{-j n\left(\varphi-\varphi_{1}\right)}}{H_{m}^{(2) \prime}(k a \sin (\theta)) H_{n}^{(2) * *}(k a \sin (\theta))} \operatorname{sinc}^{2}\left(\frac{W k \cos (\theta)}{2 \pi}\right) r^{2} \sin \theta \mathrm{d} \theta \mathrm{d} \varphi
\end{gathered}
$$

According to orthogonality property, " $m$ " is equal to " $n$ " so:

$$
\begin{aligned}
Y_{12}^{2}= & \frac{4 \alpha_{2} \alpha_{1}}{\eta \pi^{2}} \int_{c} \int_{0}^{2 \pi} \frac{\mathrm{e}^{-j k \cos (\theta)\left(z_{2}-z_{1}\right)}}{(k a \sin (\theta))^{2}}(\cot (\theta))^{2} \\
& \cdot \sum_{n=1}^{\infty} \frac{n^{2} \cos \left(\frac{n \alpha_{1}}{2}\right) \cos \left(\frac{n \alpha_{2}}{2}\right) \cos \left(n\left(\varphi-\varphi_{2}\right)\right) \cos \left(n\left(\varphi-\varphi_{1}\right)\right)}{\left[\pi^{2}-\left(n \alpha_{1}\right)^{2}\right]\left[\pi^{2}-\left(n \alpha_{2}\right)^{2}\right]\left|H_{m}^{(2) \prime}(k a \sin (\theta))\right|^{2}} \\
& \cdot \operatorname{sinc}^{2}\left(\frac{W k \cos (\theta)}{2 \pi}\right) \sin \theta \mathrm{d} \theta \mathrm{d} \varphi
\end{aligned}
$$

Modified integral path is shown in Figure 3.

The integration in term of $\varphi$ will result in (34):

$$
\begin{aligned}
Y_{12}^{2}= & \frac{8 \alpha_{2} \alpha_{1}}{\eta \pi} \int_{c} \frac{\mathrm{e}^{-j k \cos (\theta)\left(z_{2}-z_{1}\right)}}{(k a \sin (\theta))^{2}}(\cot (\theta))^{2} \\
& \cdot \sum_{n=1}^{\infty} \frac{n^{2} \cos \left(\frac{n \alpha_{1}}{2}\right) \cos \left(\frac{n \alpha_{2}}{2}\right) \cos \left(n\left(\pi^{2}-\left(n \alpha_{1}\right)^{2}\right]\left[\pi^{2}-\left(n \alpha_{2}\right)^{2}\right]\left|H_{m}^{(2)}(k a \sin (\theta))\right|^{2}\right.}{\left[\operatorname{sinc}^{2}\left(\frac{W k \cos (\theta)}{2 \pi}\right) \sin \theta \mathrm{d} \theta \mathrm{d} \varphi\right.}
\end{aligned}
$$

The integral must be divided in to real and imaginary part so for real part we have:

$$
\begin{gathered}
k_{z}=k \cos (\theta), \mathrm{d} k_{z}=-k \sin \theta \mathrm{d} \theta, \theta=\left[0, \frac{\pi}{2}\right] \rightarrow k_{z}=[k, 0] \\
Y_{12}^{2}=\frac{8 \alpha_{2} \alpha_{1}}{\eta \pi a^{2} k} \int_{0}^{k} \frac{\mathrm{e}^{-j k_{z}\left(z_{2}-z_{1}\right)}}{k^{2}-k_{z}^{2}} \frac{k_{z}^{2}}{k^{2}-k_{z}^{2}} \\
\sum_{n=1}^{\infty} \frac{n^{2} \cos \left(\frac{n \alpha_{1}}{2}\right) \cos \left(\frac{n \alpha_{2}}{2}\right) \cos \left(n\left(\varphi_{1}-\varphi_{2}\right)\right)}{\left[\pi^{2}-\left(n \alpha_{1}\right)^{2}\right]\left[\pi^{2}-\left(n \alpha_{2}\right)^{2}\right] \mid H_{m}^{(2)}\left(a \sqrt{k^{2}-k_{z}^{2}}\right)^{2}} \operatorname{sinc}^{2}\left(\frac{W k_{z}}{2 \pi}\right) \mathrm{d} k_{z} \\
\stackrel{\operatorname{I~m(\theta )}}{\longrightarrow} \underset{\operatorname{Re}(\theta)}{\longrightarrow}
\end{gathered}
$$

Figure 3. Modified integration path C. 
Now imaginary part of path must be computed. At first of hand, path must be modified i.e. for new path we have:

$$
\begin{aligned}
k_{z}= & k \cos (\theta)=k \cos (\operatorname{Re}(\theta)+j \operatorname{Im}(\theta)) \\
= & k[\cos (\operatorname{Re}(\theta)) \cosh (\operatorname{Im}(\theta))-j \sin (\operatorname{Re}(\theta)) \sinh (\operatorname{Im}(\theta))] \\
& \operatorname{Re}(\theta)=\frac{\pi}{2}, \operatorname{Im}(\theta)=[0, \infty] \Rightarrow k_{z}=[0,-j \infty]
\end{aligned}
$$

Same as above relation: $k_{z}=-j k_{z}^{\prime}, k_{z}^{\prime}=[0, \infty]$ so we have:

$$
\begin{aligned}
Y_{12}^{2}= & \frac{j 8 \alpha_{2} \alpha_{1}}{\eta \pi a^{2} k} \int_{0}^{k} \frac{\mathrm{e}^{-k_{z}^{\prime}\left(z_{2}-z_{1}\right)}}{k^{2}+k_{z}^{\prime 2}} \frac{k_{z}^{\prime 2}}{k^{2}+k_{z}^{\prime 2}} \\
& \cdot \sum_{n=1}^{\infty} \frac{n^{2} \cos \left(\frac{n \alpha_{1}}{2}\right) \cos \left(\frac{n \alpha_{2}}{2}\right) \cos \left(n\left(\varphi_{1}-\varphi_{2}\right)\right)}{\left[\pi^{2}-\left(n \alpha_{1}\right)^{2}\right]\left[\pi^{2}-\left(n \alpha_{2}\right)^{2}\right]\left|H_{n}^{(2) \prime}\left(a \sqrt{k^{2}+k_{z}^{\prime 2}}\right)\right|^{2}} \operatorname{sinch}^{2}\left(\frac{W k_{z}^{\prime}}{2 \pi}\right) \mathrm{d} k_{z}^{\prime}
\end{aligned}
$$

As a result for totality of $Y_{12}^{2}$ :

$$
\begin{aligned}
Y_{12}^{2}= & \frac{8 \alpha_{2} \alpha_{1}}{\eta \pi a^{2} k} \int_{0}^{k} \frac{\mathrm{e}^{-k_{z}\left(z_{2}-z_{1}\right)}}{k^{2}-k_{z}^{2}} \frac{k_{z}^{2}}{k^{2}-k_{z}^{2}} \\
& \cdot \sum_{n=1}^{\infty} \frac{n^{2} \cos \left(\frac{n \alpha_{1}}{2}\right) \cos \left(\frac{n \alpha_{2}}{2}\right) \cos \left(n\left(\varphi_{1}-\varphi_{2}\right)\right)}{\left[\pi^{2}-\left(n \alpha_{1}\right)^{2}\right]\left[\pi^{2}-\left(n \alpha_{2}\right)^{2}\right]\left|H_{n}^{(2) \prime}\left(a \sqrt{k^{2}+k_{z}^{\prime 2}}\right)\right|^{2}} \operatorname{sinc}^{2}\left(\frac{W k_{z}}{2 \pi}\right) \mathrm{d} k_{z} \\
& +\frac{j 8 \alpha_{2} \alpha_{1}}{\eta \pi a^{2} k} \int_{0}^{\infty} \frac{\mathrm{e}^{-k_{z}^{\prime}\left(z_{2}-z_{1}\right)}}{k^{2}+k_{z}^{\prime 2}} \frac{k_{z}^{\prime 2}}{k^{2}+k_{z}^{\prime 2}} \\
& \cdot \sum_{n=1}^{\infty} \frac{n^{2} \cos \left(\frac{n \alpha_{1}}{2}\right) \cos \left(\frac{n \alpha_{2}}{2}\right) \cos \left(n\left(\varphi_{1}-\left(n \alpha_{1}\right)^{2}\right]\left[\pi^{2}-\left(n \alpha_{2}\right)^{2}\right]\left|H_{n}^{(2) \prime}\left(a \sqrt{k^{2}+k_{z}^{\prime 2}}\right)\right|^{2}\right.}{\operatorname{sinch}^{2}\left(\frac{W k_{z}^{\prime}}{2 \pi}\right) \mathrm{d} k_{z}^{\prime}}
\end{aligned}
$$

The totality of $Y_{12}$ is sum of $Y_{12}^{1}$ and $Y_{12}^{2}$

$$
Y_{12}=Y_{12}^{1}+Y_{12}^{2}
$$

$Y_{12}$ Can be divided in to real and imaginary part:

$$
\begin{aligned}
G_{12}= & \frac{4 \alpha_{1} \alpha_{2}}{\eta \pi} \int_{0}^{k} \frac{\cos k_{z}\left(z_{2}-z_{1}\right)}{k^{2}-k_{z}^{2}} \sum_{n=0}^{\infty} \frac{\varepsilon_{n} \cos \left(\frac{n \alpha_{1}}{2}\right) \cos \left(\frac{n \alpha_{2}}{2}\right)}{\left[\pi^{2}-\left(n \alpha_{2}\right)^{2}\right]\left[\pi^{2}-\left(n \alpha_{1}\right)^{2}\right]^{2}} \\
& \cdot \frac{\cos \left(n\left(\varphi_{1}-\varphi_{2}\right)\right)}{\left|H_{n}^{(2)}\left(a \sqrt{k^{2}-k_{z}^{2}}\right)\right|} \operatorname{sinc}^{2}\left(\frac{W k_{z}}{2 \pi}\right) \mathrm{d} k_{z} \\
& +\frac{8 \alpha_{2} \alpha_{1}}{\eta \pi a^{2} k} \int_{0}^{k} \frac{\cos k_{z}\left(z_{2}-z_{1}\right)}{k^{2}-k_{z}^{2}} \frac{k_{z}^{2}}{k^{2}-k_{z}^{2}} \\
& \cdot \sum_{n=1}^{\infty} \frac{n \pi^{2} \cos \left(\frac{n \alpha_{1}}{2}\right) \cos \left(\frac{n \alpha_{2}}{2}\right) \cos \left(n\left(\varphi_{1}-\varphi_{2}\right)\right)}{\left.\left[n \alpha_{1}\right)^{2}\right]\left[\pi^{2}-\left(n \alpha_{2}\right)^{2}\right]\left|H_{n}^{(2) \prime}\left(a \sqrt{k^{2}-k_{z}^{2}}\right)\right|^{2}} \operatorname{sinc}^{2}\left(\frac{W k_{z}}{2 \pi}\right) \mathrm{d} k_{z}
\end{aligned}
$$




$$
\begin{aligned}
& B_{12}=\frac{8 \alpha_{2} \alpha_{1}}{\eta \pi a^{2} k} \int_{0}^{\infty} \frac{\mathrm{e}^{-k_{z}^{\prime}\left(z_{2}-z_{1}\right)}}{k^{2}+k_{z}^{\prime 2}} \frac{k_{z}^{\prime 2}}{k^{2}+k_{z}^{\prime 2}} \\
& \cdot \sum_{n=1}^{\infty} \frac{n^{2} \cos \left(\frac{n \alpha_{1}}{2}\right) \cos \left(\frac{n \alpha_{2}}{2}\right) \cos \left(n\left(\varphi_{1}-\varphi_{2}\right)\right)}{\left[\pi^{2}-\left(n \alpha_{1}\right)^{2}\right]\left[\pi^{2}-\left(n \alpha_{2}\right)^{2}\right]\left|H_{n}^{(2) \prime^{\prime}}\left(a \sqrt{k^{2}+k_{z}^{\prime 2}}\right)\right|^{2}} \operatorname{sinch}^{2}\left(\frac{W k_{z}^{\prime}}{2 \pi}\right) \mathrm{d} k_{z}^{\prime} \\
& +\frac{4 \alpha_{1} \alpha_{2} k}{\eta \pi} \int_{0}^{k} \frac{\mathrm{e}^{-k_{z}^{\prime}\left(z_{2}-z_{1}\right)}}{k^{2}+k_{z}^{\prime 2}} \sum_{n=0}^{\infty} \frac{\varepsilon_{n} \cos \left(\frac{n \alpha_{2}}{2}\right) \cos \left(\frac{n \alpha_{1}}{2}\right)}{\left[\pi^{2}-\left(n \alpha_{1}\right)^{2}\right]\left[\pi^{2}-\left(n \alpha_{2}\right)^{2}\right]} \\
& \cdot \frac{\cos \left(n\left(\varphi_{1}-\varphi_{2}\right)\right)}{\left|H_{n}^{(2)}\left(a \sqrt{k^{2}+k_{z}^{\prime 2}}\right)\right|^{2}} \sinh ^{2}\left(\frac{W k_{z}^{\prime}}{2 \pi}\right) \mathrm{d} k_{z}^{\prime} \\
& -\frac{4 \alpha_{1} \alpha_{2} k}{\eta \pi} \int_{0}^{k} \frac{\sin \left(k_{z}\left(z_{2}-z_{1}\right)\right)}{k^{2}-k_{z}^{2}} \sum_{n=0}^{\infty} \frac{\varepsilon_{n} \cos \left(\frac{n \alpha_{2}}{2}\right) \cos \left(\frac{n \alpha_{1}}{2}\right)}{\left[\pi^{2}-\left(n \alpha_{2}\right)^{2}\right]\left[\pi^{2}-\left(n \alpha_{1}\right)^{2}\right]^{2}} \\
& \cdot \frac{\cos \left(n\left(\varphi_{1}-\varphi_{2}\right)\right)}{\left|H_{n}^{(2)}\left(a \sqrt{k^{2}-k_{z}^{2}}\right)\right|} \operatorname{sinc}^{2}\left(\frac{W k_{z}}{2 \pi}\right) \mathrm{d} k_{z} \\
& +\frac{8 \alpha_{2} \alpha_{1}}{\eta \pi a^{2} k} \int_{0}^{k} \frac{\sin \left(k_{z}\left(z_{2}-z_{1}\right)\right)}{k^{2}-k_{z}^{2}} \frac{k_{z}^{2}}{k^{2}-k_{z}^{2}} \\
& \cdot \sum_{n=1}^{\infty} \frac{n^{2} \cos \left(\frac{n \alpha_{1}}{2}\right) \cos \left(\frac{n \alpha_{2}}{2}\right) \cos \left(n\left(\varphi_{1}-\varphi_{2}\right)\right)}{\left[\pi^{2}-\left(n \alpha_{1}\right)^{2}\right]\left[\pi^{2}-\left(n \alpha_{2}\right)^{2}\right] \mid H_{m}^{(2) '}\left(a \sqrt{k^{2}-k_{z}^{2}}\right)^{2}} \operatorname{sinc}^{2}\left(\frac{W k_{z}}{2 \pi}\right) \mathrm{d} k_{z}
\end{aligned}
$$

\section{Assessment of Parameters Effect on Convergence}

The effect of first integral of (39) on total admittance is more than effect of other integrals. Expression of (39) is strongly depended on the term of $z_{2}-z_{1}$ so that if its value increases, the convergence will not be occurred due to its cosine expression. So according to $k_{z}$ it must be noticed that the term of $z_{2}-z_{1}$ should be less than wavelength which is propagated in waveguide. Also if the cylinder radius increases so much, it can result in intense reduction of convergence, therefore radius of cylinder must be less than one $\lambda$. Susceptance has weak effect on integral totality, so limitation of parameters according to (39) must be mentioned to enhance convergence.

\section{Numerical Results}

Now we want to compare analytical results which are came from Poynting's vector method with simulation results which are yielded by Ansoft HFSS (version 13) software. For this comparison we consider expansion graph of (38) as $20 \log \left(\left|Y_{12}\right|\right)$ in terms of $\mathrm{dB}$. Integrals of graphs must be achieved by numerical methods. Upper band Value of sigma is sufficient in (15) and desired result will be achieved. Obviously if we increase upper band of sigma, better results will be 
achieved. Cylinder radius is $0.4324 \lambda$ and slot dimensions as same as each other are $0.302 \lambda \times 0.107 \lambda$. For this paper, the operation frequency is chosen $9.2 \mathrm{GHz}$ and the radius of cylinder $14.1 \mathrm{~mm}$.

In Figures 1-3 the range of admittance graph for angular offset variations of two slots is between 0 to 400 and distances between slot $z_{2}-z_{1}$ is constant and it's equal to $0.54 \lambda$.

In Figures 1-4 mutual admittance is $z_{d}=z_{2}-z_{1}$ and according to wavelength range of distance is between $0.306 \lambda$ to $1.38 \lambda$. The angular offset value (Figure 5) is $\varphi_{d}=\varphi_{1}-\varphi_{2}=30 \mathrm{deg}$. And slot aspects as same as each other are $0.302 \lambda \times 0.107 \lambda$. Because of cosine formic, the graph has some depth points. These points are periodic and summation (in those points), are negligible, therefore logarithmic form of them is consequently insignificant, in the rest of points; graph is similar to simulation result which is yielded by Ansoft HFSS software.

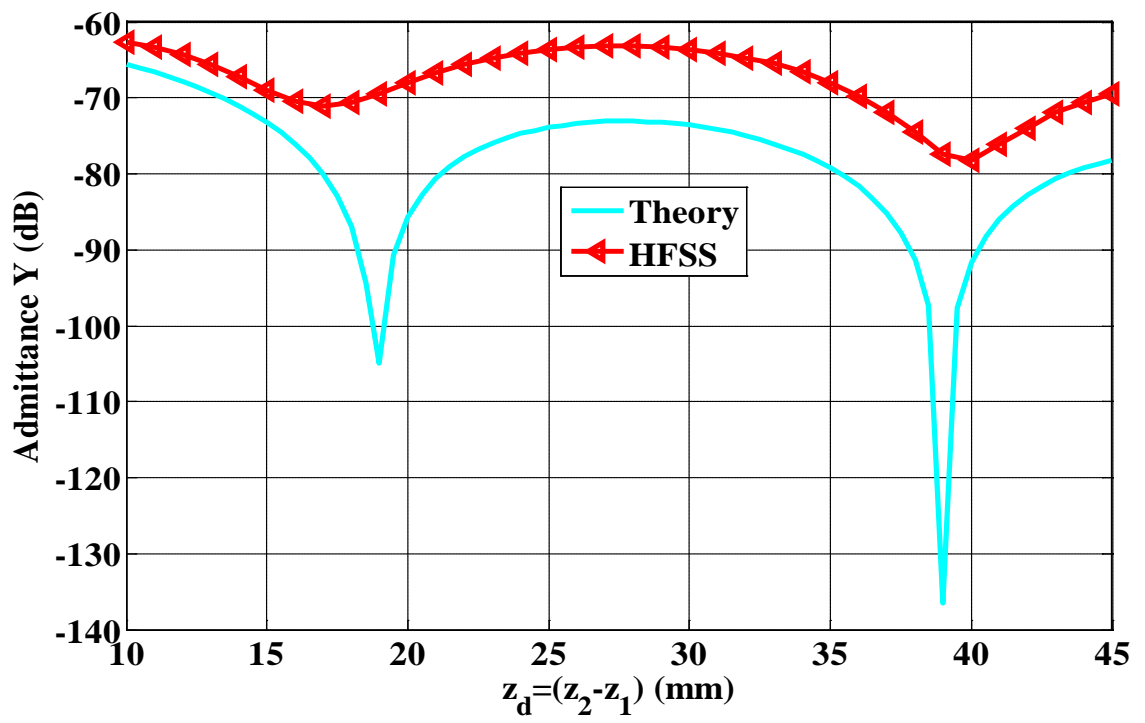

Figure 4. Admittance graph for $z_{2}-z_{1}=0.54 \lambda$.

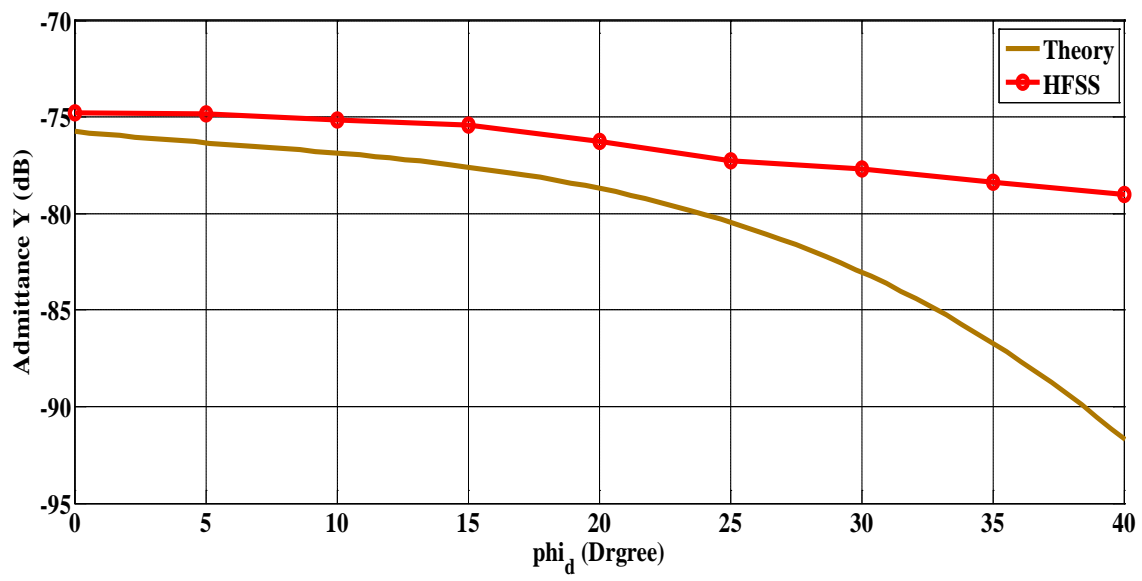

Figure 5. Admittance graph for $\varphi_{d}=\varphi_{1}-\varphi_{2}=30$ degree. 


\section{Conclusion}

In this paper mutual admittance between two circumferential slots on cylindrical waveguide by extended Poynting's vector is achieved. Analytical and simulation results are compared the results show good similarity and formulas are presented according to aforesaid limitations on parameters can be useful to find mutual admittance convergence in designing of circumferential slot antennas on cylindrical wave guide.

\section{References}

[1] Safavi-Naini, et al. (1976) Calculation of Mutual Admittance between Two Slots on a Cylinder. Attachment A In: Lee, S.W. and Mittra, R., Eds., Study of Mutual Coupling between Two Slots on a Cylinder, Electromagnetic Lab, Department of Electrical Engineering, Urbana.

[2] Golden, et al. (1974) Approximation Techniques for the Mutual Admittance of Slot Antennas on Metallic Cones. IEEE Transactions on Antennas and Propagation, 22, 43-48. https://doi.org/10.1109/TAP.1974.1140727

[3] Stewart, et al. (1971) Mutual Admittance for Axial Rectangular Slots in a Large Conducting Cylinder. IEEE Transactions on Antennas and Propagation, 19, 120-122. https://doi.org/10.1109/TAP.1971.1139875

[4] Oraizi, H., Behbahani, A.K., Noghani, M.T. and Sharafimasouleh, M. (2013) Optimum Design of Travelling Rectangular Waveguide Edge Slot Array with Non-Uniform Spacing. Journal of Microwaves, Antennas and Propagation IET, 7, 575-581. https://doi.org/10.1049/iet-map.2012.0438

[5] Masouleh, M.S. and Behbahani, A.K. (2016) Optimum Design of the Array of Circumferential Slots on a Cylindrical Waveguide. AEU-International Journal of Electronics and Communications, 70, 578-583. https://doi.org/10.1016/j.aeue.2016.01.010

[6] Azarbar, A., Masouleh, M.S. and Behbahani, A.K. (2014) A New Terahertz Microstrip Rectangular Patch Array Antenna. International Journal of Electromagnetics and Applications, 25-29.

[7] Azarbar, A., Masouleh, M.S., Behbahani, A.K. and Oraizi, H. (2012) Comparison of Different Designs of Cylindrical Printed Quadrifilar Helix Antennas. Computer and Communication Engineering (ICCCE) 2012, Kuala Lumpur, 3-5 July 2012, 18-21. https://doi.org/10.1109/ICCCE.2012.6271144

[8] Lee, et al. (1982) A Review of GTD Calculation of Mutual Admittance of Slot Conformal Array. Electromagnetics, 2, 85-127. https://doi.org/10.1080/02726348208915159

[9] Van Der Pol (1917) On the Wavelengths and Radiation of Loaded Antennas. Proceedings of the Physical Society, 29, 269-289.

[10] Hill (1967) Analytic Determination of Dipole Reactance by Radiation Pattern Integration. Proceedings of the IEEE, 114, 853-858.

[11] Sohtell (1986) Mutual Admittance between Slots on a Cylinder Using the Extended Poynting's Vector Method. Microwaves, Antennas and Propagation, IEE Proceedings $H$, 133, 238-240. https://doi.org/10.1049/ip-h-2.1986.0042

[12] Sorokosz, et al. (2010) Parametric Study of Radiation from Circumferential Slots on a Conducting Cylinder. Proceedings of 15 th Conference on Microwave Techniques, Brno, 19-21 April 2010, 31-34. https://doi.org/10.1109/COMITE.2010.5481508 